\title{
Tools for the Estimation of the Use of Agricultural Lands in the Information Systems of the Family "Constellation-VEGA»
}

\author{
Vladimir A. Tolpin ${ }^{1}$, Alexander L. Zakora ${ }^{2}$, Dmitry E. Plotnikov ${ }^{1}$, Evgeniya S. Elkina ${ }^{1}$, Alla A. \\ Kozochkina ${ }^{1}$ \\ ${ }^{1}$ Space Research Institute, Russian Academy of Sciences, Moscow, Russia \\ tolpin@d902.iki.rssi.ru \\ ${ }^{2}$ Space Research Institute for the Earth, Moscow, Russia
}

\begin{abstract}
The article is devoted to the tools and technologies for assessing the usability of agricultural lands using remote sensing data developed at IKI RAN. It describes the tools implemented in the information systems of the «Constellation-VEGA» family. Its use allows you to build maps of agricultural land utilization from small farms to levels of subjects. All work with the data takes place exclusively in the client's browser using the capabilities of the «Constellation-VEGA» family of information systems and the data archive of the Satellite Data Sharing Center - IKI Monitoring Center.
\end{abstract}

Keywords: use of agricultural land, monitoring of agricultural lands, condition of vegetation, satellite technologies of Earth observation, satellite data, remote monitoring systems, Constellation-VEGA, IKI Monitoring 


\title{
ИНСТРУМЕНТАРИЙ ОЦЕНКИ ИСПОЛЬЗУЕМОСТИ СЕЛЬСКОХОЗЯЙСТВЕННЫХ ЗЕМЕЛЬ В ИНФОРМАЦИОННЫХ СИСТЕМАХ СЕМЕЙСТВА «СОЗВЕЗДИЕ-ВЕГА»
}

\author{
В.А. Толпин ${ }^{l}$, А.Л. Закора ${ }^{2}$, Д.Е. Плотников ${ }^{1}$, Е.С. Ёлкина $^{l}$, А.А. Козочкина $^{l}$ \\ ${ }^{1}$ Институт космических исследований РАН, Москва, Россия \\ tolpin@d902.iki.rssi.ru \\ ${ }^{2}$ Институт космических исследований Земли, Москва, Россия
}

\begin{abstract}
Статья посвящена разработанным в ИКИ РАН инструментам и технологиям оценки используемости сельскохозяйственных земель с использованием данных дистанционного зондирования. В ней описывается инструментарий, реализованный в информационных системах семейства «Созвездие-ВЕГА». Его использование позволяет строить карты используемости сельскохозяйственных угодий от небольших хозяйств до уровней субъектов. Вся работа с данными происходит исключительно в браузере клиента с использование возможностей информационных систем семейства «Созвездие-Вега» и архива данных Центра коллективного пользования спутниковыми данными - ЦКП «ИКИ-Мониторинг».
\end{abstract}

Ключевые слова: использование сельскохозяйственных угодий, мониторинг $\mathrm{c} / \mathrm{x}$ земель, состояние растительного покрова, спутниковые технологии наблюдения Земли, спутниковые данные, системы дистанционного мониторинг, Созвездие-ВЕГА, ИКИ-Мониторинг

\section{Введение}

Сельскохозяйственные земли являются очень ценным природным ресурсом. От понимания насколько правильно они используются, зависит не только экономическое благополучие товаропроизводителя, но и состояние всей отрасли. Информация о частоте и правильности используемости земель позволят оценить, как состояние самих земель, так и возможность их повторного использования, а также средства и дотации, которые необходимо на это выделить.

Особенностью сельскохозяйственных земель является их площадь и структура. В России достаточно большие площади заняты под сельскохозяйственные культуры. В то же время, структуры этих площадей сильно отличаются от региона к региону. Это обуславливается как географическими особенностями, так и культивируемыми культурами. Все это приводит к тому, что размеры и формы полей могут сильно меняться. Создание полностью автоматических инструментов для решения задачи используемости земель на больших территориях пока представляется сложным из-за сильных региональных различий. Однако использование полуавтоматических инструментов и процедур может значительно облегчить труд экспертов по проведению анализа и построения карт используемости земель.

В статья описывается инструментарий информационных систем семейства «СозвездиеВЕГА», который дает возможность производить оценку используемости сельскохозяйственных земель. Его использование позволяет строить карты используемости сельскохозяйственных угодий от небольших хозяйств до уровней субъектов. Вся работа с данными происходит 
исключительно в браузере с использование возможностей Сервиса ВЕГА [1] и архива данных Центра коллективного пользования спутниковыми данными - ЦКП «ИКИ-Мониторинг» [2].

\section{Данные}

Получение актуальной оценки используемости полей невозможно без использования данных дистанционного зондирования Земли (ДЗ3). Под данными Д3З понимается не только космические снимки, но и информация, которую можно извлечь из снимков, например, вегетационные индексы. Она является постоянно актуализируемой (за счёт повторяемости съемки одного и того же участка Земли несколько раз в год), независимой, достоверной и сопоставимой между собой, что позволяет строить временные ряды данных и анализировать развитие процессов во времени. Вегетационные индексы являются относительными величинами, коррелирующими с параметрами роста растений (хлорофилл, биомасса, листовое покрытие). Источниками информации, которая используется для получения оценки являются:

- спутниковые изображения и результаты их обработки;

- тематические карты, например, карты пахотных земель или озимых (полученные по данным MODIS) [3];

- временные ряды вегетационных индексов (ВИ).

Для принятия более обоснованных решений рекомендуется использовать их в комплексе, дополняя визуальную интерпретацию анализом графиков, а также используя информацию о местности, на которой расположены оцениваемые поля.

Для визуальной оценки по спутниковым изображениям необходимо использовать данные высокого пространственного разрешения. Спутниковые изображения, получаемые с помощью сенсоров Landsat 8 и Sentinel 2 A, В являются ключевыми оценки. Они покрывают всю Россию, и их совместная частота сьемки одного и того же участка Земли составляет примерно два раза в неделю, что позволяет проанализировать историю использования поля в течение всего сезона. В качестве дополнительных источников информации можно использовать спутниковые изображения с российский спутников Канопус-В (сенсор МСС) и Ресурс-П (сенсор Геотон). Частота их получения низка, но пространственное разрешение существенно выше, чем у Landsat и Sentinel, и по этим снимкам можно оценить текстуру и структуру поля.

Для данных Landsat и Sentinel рекомендуется для анализа использовать RGB-синтез каналов сенсора OLI (Landsat-8) 6-5-4, MSI (Sentinel-2A) 11-8A-4, ETM+ (Landsat-7) 5-4-3 (дают сочетание SWIR-NIR-RED). В этом случае участки используемой пашни будут отображаться цветами от светло-розового до тёмно-фиолетового. Неиспользуемая пашня на снимках высокого пространственного разрешения имеет пятнистую текстуру. От естественного травостоя, косвенным признаком которого является приуроченность к элементам гидрографической сети, заброшенную пашню отличает схожесть с геометрией возделываемого поля. Залесённая пашня выявляется по наличию молодого древостоя с неоднородным тоном изображения, чередованием светло-зелёных и тёмно-фиолетовых полос. Также необходимо использовать зимние изображения, на которых при RGB-синтезе OLI 6-5-4 светло-красным оттенком выделяется залесённый участок. Все типы пахотных земель отображаются схожим набором оттенков на RGB-синтезе, поэтому задача их визуального детектирования требует от пользователя навыков тематического дешифрирования. В случае если принятие решения затруднительно, необходимо воспользоваться данными сверхвысокого пространственного разрешения. При визуальном анализе снимков в естественных цветах участки пашни отображаются оттенками от светлосерого до коричневого. Детектируются следы вспашки и колеи от сельскохозяйственной техники. Неиспользуемая пашня детектируется по неоднородному, пятнистому рисунку, чередованием зелёного и коричневого цветов. Залесённые участки пахотных земель легко опознаются по характерным текстурным признакам. Также для визуальной оценки по спутниковым снимкам можно использовать технологию разновременного RGB-синтеза. Для 
этого выбираются три изображения: весеннее - канал $\mathrm{R}$, летнее - канал $\mathrm{G}$ и осеннее - канал B. На этом синтезе обычно обрабатываемые поля «разноцветные», а не обрабатываемые серо, сине фиолетовые. Дополнительно можно использовать инструментарий, позволяющий проводить различные классификации на основе спутниковых данных [4].

Наряду с данными высокого и сверхвысокого разрешения можно использовать карты, полученные по данным среднего пространственного разрешения сенсора MODIS:

- карты растительного покрова;

- карты используемых пахотных земель;

- карты посевов озимых культур.

- карты земель чистого пара.

Эти карты автоматически строятся ежегодно или несколько раз в год. Использование этих карт позволяет получить дополнительную информацию о классе растительности на поле.

В том случае, когда невозможно подобрать безоблачные изображения высокого и сверхвысокого разрешения, необходимо проанализировать многолетние ряды вегетационных индексов, одним из которых является NDVI. Для построения временного ряда используется средние по полю значение характеристики (BИ NDVI) и его изменение во времени. Многолетние ряды ВИ наглядно показывают сезонные изменения биомассы. Анализ временных рядов ВИ NDVI позволяет оценить наличие севооборота на поле и восстановить историю его использования, используя данные для одного и того же поля за несколько лет. Поскольку пахотные земли - это обрабатываемые земли, подвергающиеся распашке (весной и/или осенью) и, как правило, занимаемые разными культурами из года в год (севооборот), то эти факты находят отражения на графиках NDVI. Ход вегетационного индекса на полях где производятся обработка земли отличается от полей с естественной растительностью, которые не используются. Что в ряде случаев позволяет сделать не только вывод о используемости поля, но и даже предположить какой класс растительности или культуры доминирует.

Для построения временных рядов ВИ могут использоваться любые данные доступные в системе, которые позволяют оценивать состояния растительности. Обычно это данные MODIS, Landsat или Sentinel 2 A, B. Наблюдения по данным MODIS регулярны и покрывают большие территории. Несмотря на разрешение 250м эти данные достаточно чувствительны и при анализе временных рядов позволяют получить хорошие результаты даже для небольших объектов. Они хорошо известны и достаточно однородны. Данные Landsat или Sentinel имеют уже пространственное разрешение порядка десятков метров, однако их временное покрытие пока недостаточно чтобы строить детальные временные ряды такие как для данных MODIS. Поэтому представляется наиболее рационально совместное использование данных различного разрешения при решении задач оценки в настоящее время.

\section{Инструменты}

Для работы со всеми перечисленными выше данными в информационных системах семейства «Созвездие-Вега» существует богатый набор инструментов.

Для работы со всеми видами геопространственных данных существует картографический интерфейс. Он позволяет работать со спутниковыми данными, тематическими продуктами их обработки, картами и векторными данными. Картографический интерфейс представляет собой веб-ГИС которая работает непосредственно в браузере пользователя. Он позволяет просто проводить визуальный анализ спутниковых данных и пространственное распределение различных характеристик и показателей.

Одним из типов пространственных данных является векторное покрытие границ полей. Границы полей используются для расчета пространственных характеристик поля. При расчете 
таких характеристик ее значение усредняется для всего поля. Тем самым для каждого поля получается одна цифра для характеристики для одного временного отсчета. Имея наборы таких значения для разных временных отрезков можно построить временной рад изменения характеристики.

Задать границу поля можно несколькими способами. В ручном режиме - просто нарисовать поле в интерфейсе и сохранить. Или в автоматическом режиме - импорт из файла (SHP) или использование API. В любом случае, после сохранения поля в БД для него начинается расчет временных рядов выбранных индексов (по умолчанию это NDVI). Векторное покрытие границ полей также отображается в картографическом интерфейсе, что позволяет проводить визуальный анализ.

Картографический интерфейс позволяет производить поиск, фильтрацию и выбор любых спутниковых данных доступных в архивах Центра коллективного пользования - ЦКП «ИКИМониторинг». Любые спутниковые данные можно просмотреть в различных цветовых синтезах, используя инструмент цветосинтеза. Это позволяет произвести визуальное дешифрирование данных и выявить используемые поля.

Для выделения однородных в пространстве или времени участков изображения можно воспользоваться инструментом классификации изображения. Классификация в свою очередь может быть обучаемая или необучаемая. Для формирования обучающий выборки также могут использоваться различные инструменты и информационные продукты. Из полученных однородных участков можно сформировать маску, которую затем можно пересечь с границами полей и присвоить полям класс используемости, соответствующий проценту пересечения.

Анализируя положения поля на тематически картах типов растительности можно сделать предварительный вывод о классе растительности на поле и его используемости.

Для анализа временных рядов существует специальный интерфейс. Он позволяет анализировать ход временных рядов как для выбранного объекта, так и для группы объектов, а также совместно анализировать их с такими же данными по районам или регионам. Данные для районов и регионов доступны по различным классам растительности и группам культур.

Одним из перспективных инструментов является инструмент классификации полей. Он проводит классификацию временных рядов объекта. Этот инструмент используя векторное покрытие полей, временные ряды NDVI для этих полей и предварительную обучающую выборку позволяет получить для каждого поля класс его используемости для конкретного года. Он учитывает особенности сезонной динамики спектрально - отражательных характеристик используемых пахотных земель, отличающих их от других категорий сельскохозяйственных угодий и естественной растительности. Оценка используемости пахотных земель выполняется с использованием алгоритмов машинного обучения, учитывающих особенности географического положения объектов. Для запуска процесса обучения используется база данных предварительно аннотированных полей. Количество элементов обучающей выборки может составлять небольшой процент от общего числа анализируемых объектов.

После того, как для поля определен его класс используемости его можно присвоить векторному объекту используя специальный инструмент аннотации полей. Какой именно инструмент был использован для получения это класса значения не имеет. Полю может быть присвоен один класс (в каждом сезоне): «используется», «частично используется», «не используется». После присвоения класса его всегда, в случае ошибки, можно поменять. Есть также возможно менять этот класс как для единичного объекта, так и сразу для групп объектов. Вся информации о классах сохраняется в БД и затем также используется для построения обучающих выборок.

Таким образом, для проведения анализа можно использовать следующие основные инструменты: 
- базовый функционал картографического интерфейса для поиска и просмотра спутниковых снимков;

- инструмент цветосинтеза в картографическом интерфейсе для составления произвольных RGB синтезов, в том числе разновременных;

- инструмент классификации изображения в картографическом интерфейсе, в том числе в границах полей для получения локальных карт и масок;

- интерфейс анализа временных рядов поля для изучения сезонной динамики:

- инструмент классификации полей (временных рядов) в картографическом интерфейсе для проведения полуавтоматического анализа используемости полей.

\section{Технологии}

Для оценки используемости земель и создания карты используемых полей предлагается использовать следующие технологии и инструменты. Общая схема процесса создания карты используемости земель приведена на рисунке 1.

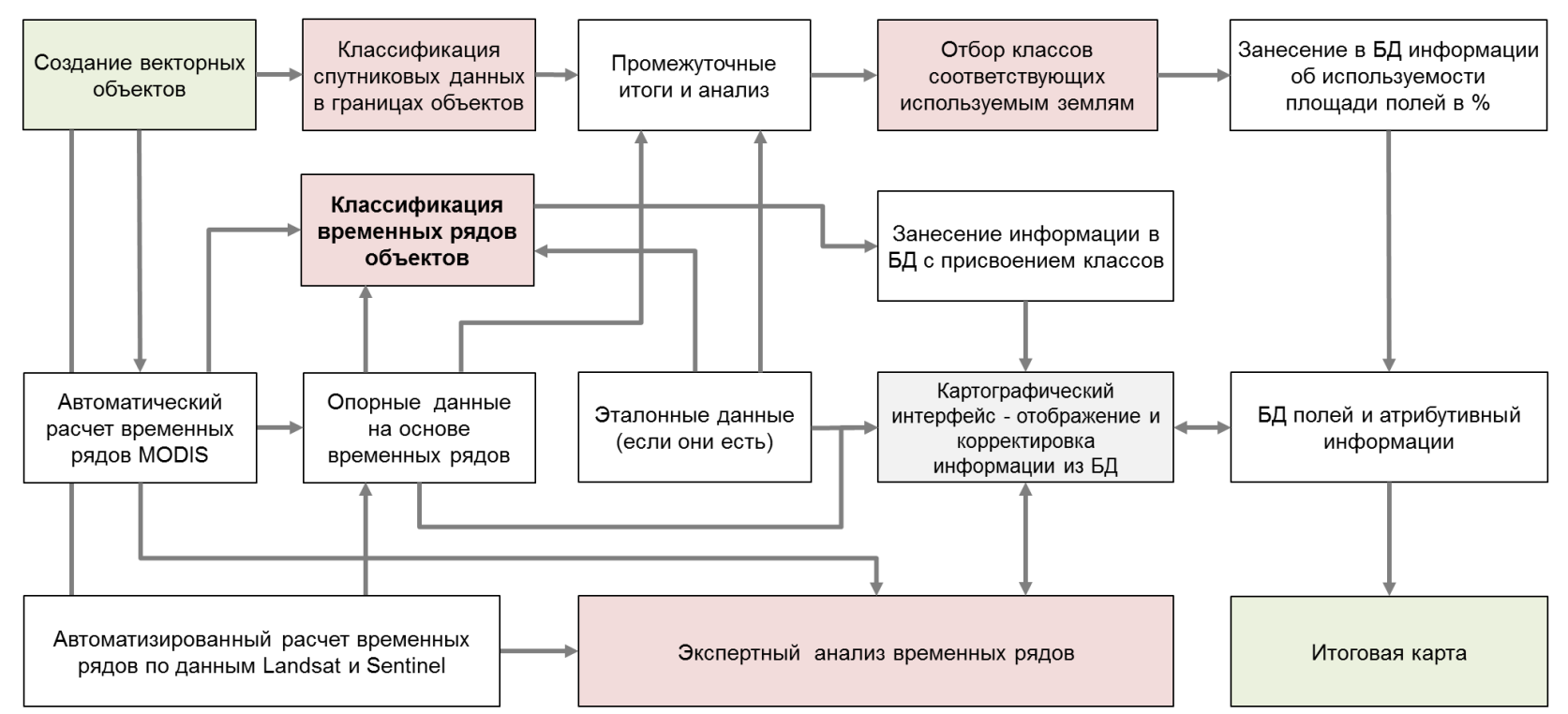

Рис. 1. Схема создания карты используемости земель

Для начала анализа используемости полей необходимо создать карту векторных границ полей. Границы полей необходимы как для визуального анализа, так и для расчета пространственно-временных характеристик, усредненных по объекту. Как только в системе появляется новый объект по нему начинается автоматический расчет временных рядов ВИ NDVI по данным MODIS и Landsat/Sentinel 2 A, B.

В зависимости от конкретной задачи можно использовать разные стратегии анализа.

Если количество объектов небольшое можно начать с экспертного анализа временных рядов. Используя данные временных рядов ВИ NDVI в ряде случаев можно сразу сделать вывод о наличие севооборота на поле и о его используемости. Если данные однозначны можно сразу присвоить класс полю, используя инструмент аннотации полей в картографическом интерфейсе. Если по данным временного ряда нельзя сделать однозначный вывод об используемости поля необходимо дополнительно провести визуальный анализ спутниковых снимков. При необходимости используя инструменты цветосинтеза и классификации.

Если требуется проанализировать значительное количество объектов имеет смысл начать с использования инструментов классификации изображения или полей. Эти инструменты 
позволять провести предварительную массовую оценку для всех выбранных полей. Используя классификацию изображения с обучением или без можно получить «гарантированные» маски используемых или неиспользуемых полей и после пересечения с векторными границами полей присвоить им соответствующий класс. Аналогично с инструментом классификации полей. Оставшиеся без классов поля или сложные случаи нужно проверять каждый отдельно используя разные инструменты и продукты:

- анализ временных рядов ВИ NDVI;

- разновременной синтез изображений;

- визуальный анализ различных стандартных продуктов и масок за разные даты.

Полученные данные по классам используемости полей в одном году можно использовать как обучающую выборку для построения карты используемых полей в другом сезоне.

Информацию о текущем статусе анализа поля можно в любой момент посмотреть, как целиком, так и по классам, а также при необходимости изменить. Можно также посмотреть статистику по выбранным объектам по классам и площадям.

После завершения аннотации полей по классам информацию можно заблокировать, подтвердив ее. В любой момент вся информация может быть выгружена из БД в виде SHP файла с соответствующими атрибутами.

\section{Заключение}

Инструментарий, реализованный в информационных системах семейства «Созвездие-Вега» с использованием возможностей архива данных Центра коллективного пользования спутниковыми данными - ЦКП «ИКИ-Мониторинг» позволяет строить карты используемости сельскохозяйственных угодий от небольших хозяйств до уровней субъектов. Вся работа с данными происходит исключительно в браузере. Имеющийся инструментарий позволяет ускорить создание карт используемых земель. Использование временных рядов вегетационного индекса позволило частично автоматизировать классификацию используемости полей.

Дальнейшие работы по развитию инструментария направлены на увеличения уровня автоматизации присвоения классов земель для облегчения построения масштабных карт за разные года.

Работа выполнена при поддержке темы «Мониторинг», госрегистрация №01.20.0.2.00164.

\section{References}

[1] Loupian E.A., Savin I.Yu., Bartalev S.A., Tolpin V.A., Balashov I.V., Plotnikov D.E. Satellite vegetation monitoring service (Vega), Sovremennye Problemy Distantsionnogo Zondirovaniya Zemli iz Kosmosa, 2011, 8(1), pp. 190-198.

[2] Loupian E.A., Proshin A.A., Burtsev M.A., Balashov I.V., Bartalev S.A., Efremov V.Yu., Kashnitskiy A.V., Mazurov A.A., Matveev A. .M., Sudneva O.A., Sychugov I.G., Tolpin V.A., Uvarov I.A. Center for collective use of systems for archiving, processing and analyzing satellite data of the IKI RAS for solving problems of studying and monitoring the environment, Sovremennye Problemy Distantsionnogo Zondirovaniya Zemli iz Kosmosa, 2015, 12(5), pp. 263-284. 
[3] Bartalev S.A., Egorov V.A., Loupian E.A., Plotnikov D.E., Uvarov I.A. Recognition of arable land based on long-term satellite data of a MODIS spectroradiometer and locally adaptive classification, Computer optics, 2011, 35(1), pp. 103-116.

[4] Kashnitskiy A.V., Balashov I.V., Loupian E.A., Tolpin V.A., Uvarov I.A. Creation of tools for remote processing of satellite data in modern information systems, Sovremennye Problemy Distantsionnogo Zondirovaniya Zemli iz Kosmosa, 2015, 12(1), pp. 156-170. 